\title{
¿ES POSIBLE UN ÍNDICE DE BANCARIZACIÓN EN ARGENTINA? UNA APLICACIÓN ESPACIAL PARA CÓRDOBA, ENTRE RÍOS Y SANTA FE ${ }^{\circ}$
}

\author{
IS AN INDEX FOR THE ONGOING BANCARIZATION \\ POSSIBLE FOR ARGENTINA? AN SPATIAL APPROACH FOR \\ CORDOBA, ENTRE RÍOS AND SANTA FE
}

Fernando García*

enviado: 6 agosto 2017 - aceptado: 21 febrero 2018

\begin{abstract}
Resumen
Este trabajo construye un Índice de Bancarización (IB) para la Argentina mediante el uso de información georreferenciada de su zona central y aplicando la metodología MULTISPATI. Los resultados muestran que las componentes espaciales seleccionadas permiten una mejor visualización de la variabilidad espacial. Este estudio constituye un aporte metodológico significativo en tanto introduce en el cálculo del IB la restricción dada por los patrones espaciales subyacentes en los datos. El IB revela un nivel de bancarización heterogéneo dentro del área central de la Argentina.
\end{abstract}

Código JEL: G20, C21, R12.

Palabras clave: índice de bancarización, datos georreferenciados,

MULTISPATI, análisis de las componentes principales.

García, F. (2018). ¿Es posible un índice de bancarización en argentina? Una aplicación espacial para córdoba, Entre Ríos y Santa Fe. Estudios económicos, 35 (70), 57-77.

* Facultad de Ciencias Económicas, Universidad Nacional de Córdoba.

Correo electrónico: fgarcia.unc@gmail.com 


\begin{abstract}
This paper constructs a Banking Index (BI) for the central area of Argentina using georeferenced data appliying MULTISPATI methodology. The results show that the selected spatial components exhibit a better visualization of the spatial variability. This study constitutes a significant methodological contribution insofar as it introduces into the $\mathrm{BI}$ compute the restriction given by the spatial patterns underlying the data. The BI reveals a heterogeneous level of banking within the main area of Argentina.
\end{abstract}

JEL Code: G20, C21, R12.

Keywords: Banking Index, Georeferenced Data, MULTISPATI, Principal Components Analysis.

\title{
INTRODUCCIÓN
}

La bancarización resulta importante en tanto constituye un motor para el desarrollo económico y social de un país o región al favorecer la disponibilidad de servicios financieros para la población y las empresas y del nivel de acceso y utilización de tales servicios por parte de los distintos agentes económicos (Grupo de Monitoreo Macroeconómico (GMM), 2011). En consecuencia, una adecuada medición del nivel y grado de disponibilidad y utilización de los servicios bancarios resulta indispensable, pues permite conocer la situación real de cada economía y facilita el diseño de medidas de política apropiadas que promuevan una mejor bancarización.

No por casualidad, la bancarización - $\mathrm{o}$, en otros términos, la inclusión financiera- se ha convertido, en los últimos años, en un objetivo importante en todos los países y en particular en la Argentina. En este sentido, se puede mencionar, como una de las iniciativas más recientes del Banco Central de la República Argentina (BCRA), la puesta en marcha del Plan Nacional de Bancarización Inclusiva 2015-2019, el cual contempla un conjunto de acciones y medidas tendientes a garantizar una mayor inclusión financiera en el país. En clara consonancia con este cometido, una medición adecuada de la evolución del proceso de bancarización a través de un Índice de Bancarización (IB) resulta clave en tanto facilita la comprensión de la bancarización y contribuye a que exista un reconocimiento respecto de su trascendencia como elemento que puede apoyar al crecimiento y desarrollo económico. 
Más allá de las características propias que le caben a la Argentina en lo que a bancarización se refiere, despierta particular interés el caso de las provincias de Córdoba, Entre Ríos y Santa Fe, las que integran la denominada Región Centro $^{1}$. Situada en el centro de la Argentina, la Región Centro posee una ubicación estratégica inmejorable para la conexión interna regional, nacional y de integración con los países del Mercado Común del Sur (MERCOSUR). Su economía es diversificada, con una producción de bienes primarios e industriales y de servicios de gran variedad, la cual tiene una importancia clave para el desarrollo socioeconómico del país. La región genera algo más del 20 \% del Producto Bruto Geográfico (PBG) argentino, ubicándose en segundo lugar luego de la región de Buenos Aires que concentra 55,5 \% del PBG. Desde el punto de vista de los servicios financieros, es de destacar que la región es la segunda en importancia, casi en un correlato directo con la participación en el PBG, en relación con la presencia de infraestructura bancaria. En efecto, información provista por el BCRA para el año 2015 da cuenta de que el número de sucursales bancarias para la región comprende $23,9 \%$ y $21,5 \%$ del total de sucursales y cajeros automáticos existentes en el país, respectivamente; sin embargo, esta misma información permite inferir que la distribución y propiedad tanto de sucursales como de cajeros dista de ser uniforme.

Bajo los preceptos anteriores, este trabajo plantea la construcción de un IB para la Región Centro de la Argentina para el año 2010, usando información georreferenciada a nivel departamental y aplicando la metodología MULTISPATI, una extensión del Análisis de Componentes Principales (PCA, son sus siglas en inglés) a datos espaciales.

El resto del trabajo se organiza como sigue. En la sección I se revisan las principales contribuciones en torno a la bancarización y a las aproximaciones empíricas que tratan de medirla. En la sección II se presenta la metodología estadística propuesta para la construcción del IB. En la sección III se presenta el IB construido a nivel departamental. Finalmente, se ensayan algunos comentarios finales.

1 La Región Centro se constituye a partir de la suscripción de tres acuerdos: el Tratado de Integración Regional (1998), el Acta de Integración de Entre Ríos (1999) y el Protocolo de Córdoba (2004), firmados todos en el marco de las facultades otorgadas a las provincias por la Constitución Nacional (Art. 124). 


\section{MARCO CONCEPTUAL}

Se considera la bancarización como "el nivel de disponibilidad, acceso y utilización de la población de un país o una región económica de los servicios ofrecidos por los bancos y/u otras entidades que forman parte del sistema financiero" (GMM, op cit.; Claessens, 2005). En coincidencia con lo anterior, el BCRA (2015) define la bancarización como el "uso masivo del sistema financiero formal por parte de los individuos para realizar transacciones financieras o económicas. Así, el concepto se relaciona con el acceso, la utilización, la calidad de los servicios financieros y el beneficio que ello genera a la población". En términos más generales, Morales y Yáñez (2006) conciben la bancarización como el establecimiento de relaciones estables y amplias entre las instituciones financieras y sus usuarios respecto de un conjunto de servicios financieros disponibles.

Mayores niveles de bancarización promueven una mayor inclusión financiera. En un sentido amplio, la inclusión financiera puede definirse como el proceso que garantiza la accesibilidad, disponibilidad y uso del sistema financiero formal para todos los miembros de una economía (Chakravarty y Pal, 2013; Sarma, 2008). En esta dirección y en consonancia con los lineamientos del Banco Mundial, el BCRA entiende la inclusión financiera como el acceso y el uso de una oferta amplia de servicios financieros, provistos de una manera sostenible y responsable. En este trabajo se utilizará el término bancarización como análogo al de inclusión financiera.

Existe una amplia literatura empírica que analiza la bancarización. Entre los trabajos pioneros, pueden mencionarse a Jacobs (1965) y Lanzilotti y Saving (1969), cuyas investigaciones se han focalizado en los efectos de la desregulación en los Estados Unidos sobre la accesibilidad a los servicios bancarios. En esta misma línea se inscriben los trabajos de Savage y Humphrey (1979), Seaver y Fraser (1979, 1983) y Evanoff (1988), quienes, aunque utilizando las mismas metodologías, sugieren algunos cambios tanto en la definición de la variable utilizada para medir la accesibilidad a los servicios bancarios como en la unidad geográfica de análisis.

En términos generales, sin embargo, la literatura ha abordado el fenómeno de la bancarización a través de estudios que buscan analizar sus determinantes. En el caso de América Latina, en particular cabe mencionar los estudios para Brasil (Kumar, 2005), Colombia (Medina y Núñez, 2006) y México (Castellanos et al., 2009).

En el caso argentino, a pesar de la importancia del fenómeno y de su impacto sobre la economía, la literatura es aun relativamente novel. Al respecto, corres- 
ponde mencionar el trabajo de Anastasi et al. (2010), quienes manifiestan que —en ausencia de datos específicos sobre bancarización provenientes de encuestas que permitan cuantificar el nivel de acceso y utilización real de los servicios bancarios- la disponibilidad de agencias bancarias (sucursales y cajeros automáticos) y el nivel de depósitos y préstamos, como indicadores alternativos, constituyen una aproximación razonable.

Sin embargo, si bien es vasta la literatura empírica que estudia la bancarización, la construcción de un índice de bancarización no ha recibido demasiada atención en la literatura, siendo prácticamente nulos los trabajos para la Argentina. Pese a esta situación, existen antecedentes interesantes respecto de índices de este tipo.

Cámara y Tuesta (2014) plantean que la medición multidimensional de la inclusión financiera resulta importante por varias razones. Una medida que agrega varios indicadores en un solo índice ayuda a resumir la naturaleza compleja de la inclusión financiera. Por otro lado, monitorear diferentes indicadores individualmente, aunque útil, no ofrece una comprensión integral del fenómeno. Adicionalmente, una medida de este tipo permite estudiar la relación entre la inclusión financiera y otras variables macroeconómicas de interés. Asimismo, la disponibilidad de información de cada dimensión ayuda a comprender mejor el fenómeno de interés. Finalmente, una medida de este tipo resulta una herramienta útil para la formulación y evaluación de políticas.

Existen dos enfoques comúnmente utilizados para la construcción de índices compuestos. Un primer enfoque asigna los pesos relativos de cada indicador sobre el índice global en forma exógena. El segundo enfoque, en cambio, sostiene que tales ponderadores deben determinarse endógenamente a partir de la estructura de covariación de los indicadores considerados.

Dentro del primer enfoque, se pueden mencionar los trabajos de Sarma (2008, 2012), Arora (2010), Gupte et al. (2012), Chakravarty y Pal (2013), Hasan e Islam (2016) y Sethy (2016), entre otros.

Sarma (2008) sugiere un índice de inclusión financiera utilizando el enfoque multidimensional adoptado para el cálculo del Índice de Desarrollo Humano (IDH) del Programa de las Naciones Unidas para el Desarrollo (PNUD), pero basado en una medida de distancia respecto de la situación ideal. El índice propuesto considera un indicador simple en cada una de las tres dimensiones básicas: penetración (o accesibilidad), disponibilidad y uso. En la misma dirección, Arora (2010) utiliza esta metodología, pero incorporando indicadores múltiples en las 
dimensiones de análisis: alcance (penetración y accesibilidad), facilidad de las transacciones y costo de las transacciones; y propone diferentes ponderadores para tales dimensiones.

Gupte et al. (2012) construyen un índice de inclusión financiera para la India utilizando el enfoque adoptado para el cálculo del IDH del PNUD, pero aplicando la media geométrica para el cómputo del índice global, el que considera cuatro dimensiones básicas: alcance (penetración y accesibilidad), uso, facilidad de las transacciones y costo de las transacciones. Para el mismo país, Chakravarty y Pal (2013) diseñan un índice aplicando una aproximación axiomática que mejora la medida propuesta por Sarma (2008) en tanto permite determinar la contribución relativa de cada una de las dimensiones consideradas.

En un trabajo posterior, Sarma (2012) introduce una mejora en el índice propuesto originalmente considerando en su cálculo no solo la distancia respecto del ideal sino también de la situación peor. En la misma dirección, Hasan e Islam (2016) proponen un índice de inclusión financiera para Bangladesh siguiendo la metodología propuesta por Sarma (2012). Utilizando idéntica metodología, Sethy (2016) elabora un índice de inclusión financiera para la India y considera indicadores tanto de demanda como de oferta del sistema financiero.

En el segundo enfoque se inscriben los trabajos de Zulaica Piñeyro (2013), Amidzic et al. (2014), Cámara y Tuesta (2014) y Mishra et al. (2014), entre otros.

Zulaica Piñeyro (2013) construye un índice de inclusión financiera para México considerando las dimensiones acceso y uso de servicios financieros, educación financiera, protección al consumidor y desarrollo social a través del PCA.

Amidzic et al. (2014) proponen un índice compuesto que utiliza el análisis factorial para identificar las dimensiones de la inclusión financiera y determinar los pesos relativos de cada una sobre el índice global. Cámara y Tuesta (2014), en cambio, empleando PCA en dos etapas (ya que consideran que es más adecuado que el análisis factorial), proponen una medida integral de inclusión financiera incorporando como dimensiones de análisis el uso, las barreras y el acceso.

Finalmente, Mishra et al. (2014) construyen un índice de inclusión financiera para la India utilizando PCA y proponen distintos métodos para asignar los pesos relativos de cada dimensión de análisis sobre el índice global. 
El antecedente más importante para Latinoamérica como sugerencia hacia un avance en este sentido lo constituye el documento del GMM (2011), el cual propone un indicador compuesto de bancarización para los países miembros del MERCOSUR. Este trabajo presenta dos metodologías para la construcción del indicador: PCA y Doing Business Adaptada ${ }^{2}$. Aunque interesante, en tanto ambas propuestas posibilitan una medición del fenómeno de manera global y sintética, dichos indicadores podrían verse afectados por los patrones espaciales subyacentes en los datos que resultan ignorados por ambas. Es decir, ninguna de las dos metodologías considera la dimensión espacial del fenómeno.

Bajo los preceptos anteriores, este trabajo propone un índice de bancarización que introduce en su cálculo la restricción dada por los datos georreferenciados, constituyendo, por tanto, un aporte metodológico significativo.

\section{METODOLOGÍA}

Para la construcción del IB, se considera como punto de partida el $\mathrm{PCA}^{3}$.

La utilización de esta metodología se ha extendido especialmente en los casos en que no existe un consenso entre los expertos sobre la importancia relativa de las variables, puesto que de forma interna proporciona un mayor peso a las variables más altamente correlacionadas con el conjunto de variables restantes en el sistema. (Domínguez et al., 2011, p. 47).

La elección del PCA fue motivada, adicionalmente, por la posibilidad de incorporar información espacial en la construcción de dicho índice constituyendo, de esta manera, una propuesta original para la medición del nivel de bancarización.

2 Los indicadores de Doing Business son construidos a partir de la creación de escenarios estandarizados de acuerdo con supuestos específicos. Estos escenarios estandarizados son utilizados como benchmarks para medir cada uno de los indicadores considerados y contrastarlos con las condiciones reales de cada una de las economías analizadas en el estudio. Para mayores detalles sobre esta metodología, se puede consultar Djankov et al. (2005).

3 El PCA es una técnica estadística multivariada que permite identificar las variables que explican la mayor parte de la variabilidad total contenida en los datos, explorar las correlaciones entre variables y reducir la dimensión del análisis con la menor pérdida de información posible, al combinar todas las variables en nuevos índices (variables sintéticas). Cada una de estas nuevas variables constituye una componente principal (Peña, 2002). Estas componentes son combinaciones lineales ortogonales (independientes) de las variables originales y se espera que solo unas pocas (las primeras) recojan la mayor parte de la variabilidad de los datos, obteniéndose una reducción de la dimensión en los mismos. 
Cuando los datos están georreferenciados, es decir asociados con una localización geográfica, es importante remarcar que la estructura de covariación reflejada por un análisis multivariado clásico (PCA en este trabajo) puede verse afectada por los patrones espaciales subyacentes en los datos. La incorporación de la información geográfica puede realizarse a posteriori del PCA mediante la asignación de los valores de las componentes a cada uno de los sitios georreferenciados o ajustando semivariogramas (Schabenberger y Pierce, 2002). La presencia de autocorrelación espacial en las Componentes Principales (CPs) también se puede detectar utilizando el estadístico I de Moran (Moran, 1950) o el C de Geary (Geary, 1954).

Dray et al. (2011) proponen un método de análisis multivariado que incorpora la información espacial antes del análisis multivariado, conocido como MULTISPATI. Constituye una extensión del PCA a datos espaciales, es decir se basa en el PCA pero incorpora la restricción dada por los datos espaciales mediante el cálculo del índice de Moran para medir la autocorrelación espacial entre las observaciones, para lo cual es necesario definir una matriz de pesos espaciales denominada $\mathrm{W}^{4}$. MULTISPATI maximiza el producto entre la varianza y la autocorrelación espacial de las CPs, mientras que PCA maximiza solo la varianza.

Yendo específicamente al cometido de este trabajo, en el proceso de construcción del IB se sigue a Nardo et al. (2008). El punto de partida de este procedimiento es la definición de un marco conceptual adecuado, que permitirá definir las dimensiones del fenómeno a estudiar. Siguiendo la propuesta del GMM (2011), se tienen en cuenta tres dimensiones de análisis: "Magnitudes Agregadas" (se consideran los aspectos macroeconómicos o medidas globales de bancarización, como elementos que dan una idea general del tamaño del sistema financiero), "Disponibilidad y Cobertura Geográfica" (para medir la capilaridad del sistema financiero a través de la existencia o no de sucursales y cajeros automáticos) y "Acceso y Utilización" (que permiten medir el grado de utilización de los servicios financieros) $)^{5}$.

4 Este análisis permite estudiar las relaciones entre las variables medidas (análisis covariabilidad) y, al mismo tiempo, la estructura espacial (autocorrelación).

5 Resulta pertinente aclarar que los indicadores usados para esta última dimensión se refieren a la utilización y no al acceso, es decir constituyen aproximaciones a este último, dada la imposibilidad de disponer de datos de acceso a los servicios financieros propiamente dichos, los cuales podrían obtenerse solamente mediante encuestas. Asimismo, además de las dimensiones mencionadas, existen otros factores que limitan o restringen el nivel o grado de bancarización. Estos condicionamientos en muchos casos se refieren a aspectos legales, barreras culturales, tecnológicas y de costos de transacción que deberían ser considerados. 
Una vez definido el marco conceptual, se procede a la selección de los indicadores parciales que se utilizan para medir cada una de las dimensiones consideradas. Esta etapa constituye un primer proceso de validación de la utilidad de los indicadores seleccionados, ya que supone verificar su pertinencia para estimar el comportamiento de las dimensiones elegidas para su estudio.

En este trabajo, se utilizan los indicadores propuestos por el GMM para la construcción del Indicador Único de Bancarización MERCOSUR. El primer grupo de indicadores considerado se refiere a aquellos que capturan la bancarización desde el punto de vista de las "Magnitudes Agregadas". Aunque los indicadores propuestos son los Depósitos del Sector Privado como proporción del PIB y los Créditos al Sector Privado como proporción del PIB, debido a que no está disponible el PIB desagregado a nivel departamental (a excepción de la provincia de Córdoba), se adoptó una definición alternativa del indicador (también sugerida por GMM): el Saldo de Depósitos [DEPPOB] y Préstamos [PREPOB] al Sector Privado en referencia a la población total ${ }^{6}$.

Respecto de la "Disponibilidad y Cobertura Geográfica", los indicadores propuestos capturan información sobre el número de sucursales y cajeros automáticos. Dentro de los indicadores que procuran medir la cobertura geográfica media o global de servicios financieros, se proponen el Número de Sucursales [SUCKM2] y Cajeros Automáticos [CAJKM2] por 1000 kilómetros cuadrados. La disponibilidad media de tales servicios es capturada, en cambio, a través del Número de Sucursales y Cajeros Automáticos cada 100.000 habitantes.

El último grupo de indicadores refiere a la "Utilización y Acceso" al sistema financiero. Los indicadores propuestos son el Número de Cuentas Bancarias, Tarjetas de Débito y Crédito cada 100.000 habitantes. Aunque dicha información está disponible, solo es de acceso público agregada a nivel de país y no a nivel departamental, motivo por el cual no se utilizó. Cabe aclarar que estos indicadores son más bien "proxies" de los de acceso. Como ya se indicó oportunamente, información precisa de acceso solamente puede ser obtenida a través de encuestas. En su lugar, se utiliza la Proporción de Población con Cobertura de Servicios Bancarios [COB], es decir que vive en localidades que cuentan con infraestructura

6 Si bien los indicadores de magnitudes agregadas también pueden ser vistos como indicadores de utilización, existe un consenso generalizado en considerarlos en una categoría separada, debido a su referencia al PIB. Por otra parte, en los indicadores de utilización y acceso se focaliza el análisis en las personas. De allí que, debido a la definición alternativa utilizada, bien podría incluirse en la última dimensión de análisis. 
bancaria (presencia de sucursal y/o cajero), y la Proporción de Localidades del Departamento que cuenta con Infraestructura Bancaria [COBLOC $]^{7}$. Aunque no miden la utilización efectiva de los servicios financieros, estos indicadores constituyen aproximaciones adecuadas para medir el acceso.

Cabe aclarar que el trabajo no ignora la existencia de otros canales por los cuales la bancarización se generaliza actualmente, tal el caso del uso de redes o Internet, cuyos datos son difíciles de obtener y procesar por una cuestión de costos y metodología, y que quedarán para ulteriores análisis. Aun así, siempre será necesario un primer contacto con la institución financiera y, en este sentido, la presencia de la tradicional sucursal bancaria deberá dar el puntapié inicial en la vinculación del cliente con el servicio, esto es su "socialización" inicial con el sistema financiero.

Resulta importante mencionar que en el proceso de selección de los indicadores se han considerado también aspectos adicionales, los cuales refieren a que los mismos deben poder ser calculados regularmente, a partir de información proporcionada por una autoridad confiable, de acuerdo a estándares y que se encuentren disponibles para el público en general.

Para la construcción de los indicadores se utilizó información de la infraestructura bancaria disponible (sucursales bancarias y cajeros automáticos) y de los préstamos y depósitos al sector privado correspondientes a diciembre de 2010 (BCRA, 2010). Los datos de la población se obtuvieron a partir del Censo Nacional de Población, Hogares y Viviendas 2010 (INDEC, 2010). Si bien existen datos más actualizados sobre el sistema financiero, los últimos datos disponibles referidos a población, tanto a nivel de localidad como departamental, corresponden al año 2010.

Como los indicadores seleccionados están medidos en distintas escalas, se hace necesario un proceso de normalización para que puedan ser agregados de manera comparable. Nardo et al. (2008) sugieren varios métodos. En este trabajo, se considera el procedimiento de estandarización de los datos, el cual convierte el indicador a una escala común con media cero y desviación estándar uno ${ }^{8}$.

7 Si bien estos indicadores son sugeridos por GMM (2011), no fueron utilizados en la construcción del indicador de bancarización para el MERCOSUR debido a que no estaban disponibles para todos los países que compara en el estudio.

8 Este procedimiento está implícito cuando se aplica PCA y se trabaja a partir de la matriz de correlación de los datos. 
Domínguez et al. (2011) hacen una revisión de la literatura existente distinguiendo distintas metodologías que resultan útiles para agregar la información disponible en una medida global única. En este trabajo, como se señaló anteriormente, se utiliza MULTISPATI. Es decir, el indicador global se define a partir de la agregación de los valores de los indicadores parciales seleccionados ${ }^{9}$.

$$
\begin{array}{r}
\operatorname{Ind}_{i}=\sum_{j=1}^{p} \alpha_{j} \cdot Z_{i j} \\
\alpha_{j}=\frac{\sum_{k=1}^{p} a_{j k} \cdot \lambda_{k}}{\sum_{j=1}^{p} \sum_{k=1}^{p} a_{j k} \cdot \lambda_{k}}
\end{array}
$$

donde $I n d_{i}$ es el valor del indicador para la $i$-ésima unidad, $a_{j}$ es el peso relativo del $j$-ésimo indicador parcial, $Z_{i j}$ es el valor estandarizado del $j$-ésimo indicador parcial para la $i$-ésima unidad, $\lambda_{k}$ es el autovalor asociado a la $k$-ésima $C P$ y $a_{j k}$ es el factor de peso correspondiente al $j$-ésimo indicador parcial en la $k$-ésima $C P$.

Finalmente se decidió transformar el valor del indicador a una escala [0100] para facilitar su interpretación ${ }^{10}$.

$$
I B_{i}=\frac{\operatorname{Ind} d_{i}-\min \left(\operatorname{Ind} d_{i}\right)}{\operatorname{má} x\left(\operatorname{Ind} d_{i}\right)-\min \left(\operatorname{Ind} d_{i}\right)} .100
$$

Para realizar los análisis estadísticos se utilizó el software libre R (www.r-project.org), siendo empleadas las librerías ade4 (Chessel et al., 2004) y spdep (Bivand et al., 2010).

9 El uso de los autovalores asociados a cada CP como ponderación garantiza la mayor incidencia o importancia en el indicador global de aquellas CPs que explican una mayor porción de la variabilidad total de los datos.

10 Este procedimiento permite escalar el rango original de los datos al rango $[0,100]$ donde el 100 indica el mayor nivel de bancarización y 0 el menor. 


\section{RESULTADOS}

El análisis descriptivo previo de los indicadores parciales permitió detectar la presencia de valores extremos en los indicadores Número de Sucursales y Cajeros Automáticos cada 1000 kilómetros cuadrados, por lo que su inclusión en el análisis podría introducir distorsiones en la construcción del IB. Por tal motivo se decidió realizar una transformación logarítmica a tales indicadores ([LNSUCKM2] y [LNCAJKM2] respectivamente). A modo ilustrativo, la tabla 1 muestra los estadísticos descriptivos de los indicadores parciales utilizados en la construcción del IB, calculados para el año 2010.

Tabla 1. Descriptivas de los indicadores parciales de bancarización

\begin{tabular}{lcccc}
\hline Indicador & Media & Coeficiente de variación (\%) & Mínimo & Máximo \\
\hline$[$ DEPPOB] & 2,77 & 57,9 & 0,52 & 6,23 \\
{$[$ PREPOB] } & 2,19 & 57,2 & 0,49 & 4,66 \\
{$[$ LNSUCKM2] } & 0,47 & 287,7 & $-2,32$ & 5,26 \\
{$[$ LNCAJKM2] } & 1,01 & 164,9 & $-2,32$ & 6,94 \\
{$[$ SUCPOB] } & 14,41 & 49,7 & 4,79 & 37,59 \\
{$[$ CAJPOB $]$} & 24,08 & 41,3 & 6,46 & 53,26 \\
{$[$ COB $]$} & 81,26 & 18,2 & 32,12 & 100,00 \\
{$[$ COBLOC $]$} & 37,81 & 56,2 & 11,11 & 100,00 \\
\hline
\end{tabular}

Fuente: elaboración propia.

En forma complementaria, se analizó la distribución espacial de dichos indicadores a través de mapas de coropletas ${ }^{11}$. Si bien la observación de los mapas permite captar de forma intuitiva la existencia de patrones espaciales, resulta fundamental contar con criterios estadísticos objetivos, el estadístico I de Moran en nuestro caso, capaces de detectar la presencia significativa de autocorrelación espacial ${ }^{12}$.

11 El mapa constituye un elemento central dentro del Análisis Exploratorio de Datos Espaciales (AEDE) que permite explorar los datos espaciales, detectar patrones y formular hipótesis que se refieren a la presencia de fenómenos espaciales dentro de los datos e identificar casos atípicos dada su localización en el mapa, entre otros (Acevedo y Velásquez, 2008).

12 Estos criterios estadísticos contrastan la hipótesis de que una variable se encuentre distribuida aleatoriamente o si, por el contrario, existe algún tipo de asociación significativa de valores similares o distintos en regiones vecinas. 
Posteriormente, se procedió a su agregación en un solo indicador global, aplicando PCA. Debido a que las variables originales están en distintas unidades y presentan distintas escalas de medida se decidió trabajar con la matriz de correlaciones (Peña, 2002). Aunque una práctica común es considerar solo las primeras CPs, en tanto permiten explicar la mayor parte de la variabilidad de los datos (García, 2017) ${ }^{13}$, en este trabajo se sigue la sugerencia de Cámara y Tuesta (2014) de incorporar en el cálculo del indicador todas las CPs ya que el objetivo final no es reducir la dimensionalidad de los datos sino mejorar la precisión en la medición.

Para el cálculo del Índice de Moran se utilizó la matriz de pesos espaciales W. Para su construcción se consideró un criterio combinado de distancia y contigüidad y para ello se usó un umbral que permite reducir la conectividad entre todas las regiones y que pondera por la inversa de la distancia a las regiones vecinas siendo el promedio de vecinos similar al obtenido por contigüidad. En particular, se utiliza como punto de referencia al centroide de cada departamento y considera vecinos aquellos departamentos ubicados a menos de $104 \mathrm{~km}^{14}$. En el gráfico 1 se muestra el mapa de contactos o vecindad de acuerdo con el criterio utilizado.

La disponibilidad de datos georreferenciados motivó la aplicación de técnicas que incorporen la información espacial, lo cual se logró mediante la implementación de la metodología MULTISPATI. De hecho, la presencia de autocorrelación espacial significativa en los indicadores parciales utilizados (ver tabla 2) confirmó la existencia de patrones espaciales subyacentes en los datos en la estructura de covariación reflejada por un análisis multivariado clásico. Para ello se introdujo la matriz de pesos espaciales $\mathrm{W}$ en el PCA de la matriz de datos originales, permitiendo estudiar las relaciones entre las variables medidas $\mathrm{y}$, al mismo tiempo, la estructura espacial.

13 En García (2017) se consideraron dos CPs, siguiendo la regla de conservar aquellas con valor propio mayor a la unidad, debido a que se trabajó con la matriz de correlación. Esta regla coincide con el criterio de recoger unas pocas componentes que permitan explicar la mayor parte de la variabilidad de los datos (Peña op. cit.).

14 En esta aplicación, este umbral coincide con la mínima distancia que garantiza que todos los departamentos tengan al menos un vecino. Adicionalmente, se decidió trabajar con una transformación de dicha matriz (normalización por fila) ya que permite mejorar las propiedades estadísticas de los estimadores y sus estadísticos (Herrera et al., 2012). 
Gráfico 1. Región Centro: mapa de vecindad

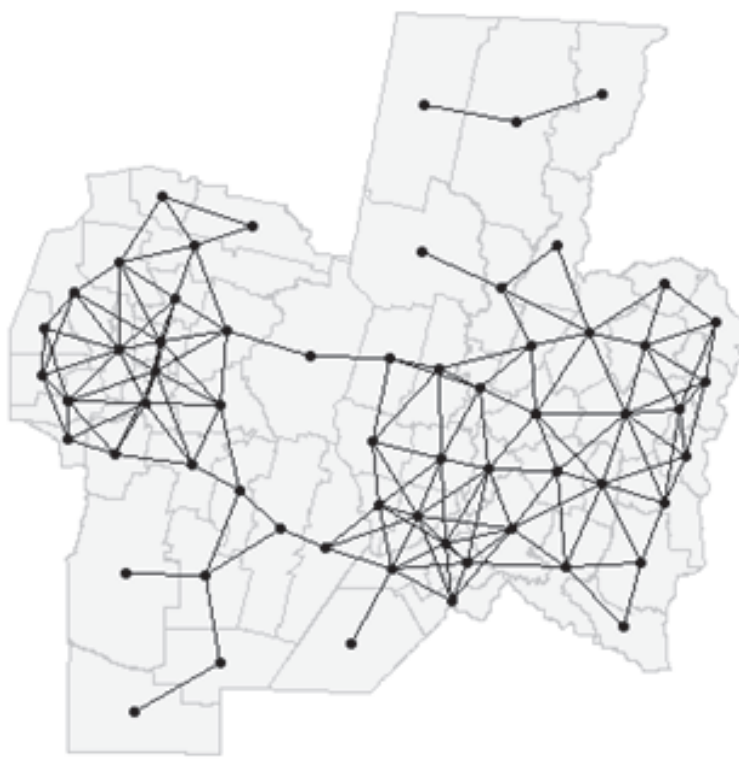

Fuente: elaboración propia.

Tabla 2. Indicadores parciales - I de Moran

\begin{tabular}{lc}
\hline Indicador & I Moran \\
\hline$[\mathrm{DEPPOB}]$ & $0,491 * * *$ \\
{$[\mathrm{PREPOB}]$} & $0,569 * * *$ \\
{$[\mathrm{LNSUCKM}]$} & $0,427 * * *$ \\
{$[\mathrm{LNCAJKM}]$} & $0,384 * * *$ \\
{$[\mathrm{SUCPOB}]$} & $0,424 * * *$ \\
{$[\mathrm{CAJPOB}]$} & $0,349 * * *$ \\
{$[\mathrm{COB}]$} & $0,547 * * *$ \\
{$[\mathrm{COBLOC}]$} & $0,494 * * *$ \\
\hline
\end{tabular}

Nota: $(* * *)$ significativo al $1 \%$.

Fuente: elaboración propia. 
A modo ilustrativo, en las tablas 3 y 4 se presentan las varianzas y los coeficientes de autocorrelación espacial (I de Moran) para las cuatro primeras CPs generadas a partir de PCA y MULTISPATI, respectivamente.

Los resultados muestran que si bien las CPs que obtiene el PCA espacial explican una menor proporción de la varianza acumulada en las cuatro primeras CPs, en esta aplicación, respecto de PCA (88,2 \% vs. 92,7\%), la variabilidad que explican es aquella con mayor estructura espacial. Esto puede observarse mediante los valores del índice de Moran calculados para tales CPs, los cuales sugieren que la estimación de autocorrelación espacial aumentó cuando se usó MULTISPATI respecto de la contenida en las CPs del PCA. Este resultado permitiría una visualización mejor de la variabilidad espacial y corroborar que PCA espacial resulta más adecuado, en esta aplicación, en relación con PCA. De esta manera, sería recomendable avanzar en la construcción del IB a partir de las CPs obtenidas a través de MULTISPATI.

Tabla 3. Análisis de componentes principales

\begin{tabular}{ccccc}
\hline Componente & $\begin{array}{c}\text { Autovalores } \\
\text { (varianza) }\end{array}$ & $\begin{array}{c}\text { Proporción } \\
\text { (varianza) }\end{array}$ & $\begin{array}{c}\text { Proporción } \\
\text { acumulada }\end{array}$ & Índice de Moran \\
\hline 1 & 5,277 & 0,660 & 0,660 & $0,529 * * *$ \\
2 & 1,273 & 0,159 & 0,819 & $0,404 * * *$ \\
3 & 0,446 & 0,056 & 0,875 & $0,504 * * *$ \\
4 & 0,420 & 0,053 & 0,927 & 0,041 \\
\hline
\end{tabular}

Nota: $(* * *)$ significativo al $1 \%$.

Fuente: elaboración propia.

Tabla 4. Análisis de componentes principales espaciales

\begin{tabular}{cccccc}
\hline Componente & Autovalores & $\begin{array}{c}\text { Varianza } \\
\text { espacial }\end{array}$ & $\begin{array}{c}\text { Proporción } \\
\text { (varianza } \\
\text { espacial) }\end{array}$ & $\begin{array}{c}\text { Proporción } \\
\text { acumulada }\end{array}$ & $\begin{array}{c}\text { Índice de } \\
\text { Moran }\end{array}$ \\
\hline 1 & 2,861 & 5,158 & 0,645 & 0,645 & $0,555 * * *$ \\
2 & 0,531 & 1,236 & 0,154 & 0,799 & $0,430 * * *$ \\
3 & 0,228 & 0,442 & 0,055 & 0,854 & $0,517 * * *$ \\
4 & 0,088 & 0,217 & 0,027 & 0,882 & $0,407 * * *$ \\
\hline
\end{tabular}

Nota: $(* * *)$ significativo al $1 \%$.

Fuente: elaboración propia. 
De acuerdo con lo establecido en la sección anterior, se definió el indicador global a partir de la agregación de los valores de los indicadores parciales propuestos. En la tabla 5 se muestran los pesos relativos correspondientes a los indicadores parciales obtenidos a partir de PCA y MULTISPATI. Finalmente, se transformó el valor del indicador a una escala [0-100].

Tabla 5. Pesos relativos de los indicadores parciales

\begin{tabular}{lcc}
\hline Indicador & PCA & MULTISPATI \\
\hline$[\mathrm{DEPPOB}]$ & 0,1428 & 0,1481 \\
{$[\mathrm{PREPOB}]$} & 0,1704 & 0,1816 \\
{$[\mathrm{LNSUCKM}]$} & 0,1014 & 0,0873 \\
{$[\mathrm{LNCAJKM} 2]$} & 0,0850 & 0,0574 \\
{$[\mathrm{SUCPOB}]$} & 0,1300 & 0,1430 \\
{$[\mathrm{CAJPOB}]$} & 0,1565 & 0,1158 \\
{$[\mathrm{COB}]$} & 0,1106 & 0,1258 \\
{$[\mathrm{COBLOC}]$} & 0,1033 & 0,1410 \\
\hline
\end{tabular}

Fuente: elaboración propia.

El gráfico 2 muestra la distribución espacial del IB departamental para la Región Centro para el año 2010 generado a partir de PCA espacial. A efectos de definir el nivel de bancarización se consideraron los siguientes intervalos: $[0,20]$ Bajo, [20,40] Medio-Bajo, [40,60] Medio, [60,80] Medio-Alto y [80,100] Alto. Según se puede observar, solo para tres departamentos la clasificación según la escala anterior comparando PCA y PCA espacial no coincidió: Juárez Celman, Marcos Juárez y Río Segundo Fe (todos de Córdoba), siendo en los tres casos el nivel de bancarización mayor teniendo en cuenta PCA espacial.

Del gráfico se infiere que la provincia de Santa Fe es la que presenta un mayor nivel de bancarización. Solo 4 de los 19 departamentos poseen niveles Bajo y Medio-Bajo, perteneciendo el resto mayoritariamente al nivel Medio-Alto. Sigue en importancia la provincia de Córdoba, pero con un comportamiento más heterogéneo (mayor coeficiente de variación) que contrasta con el de la provincia de Entre Ríos, que, aunque presenta un nivel de bancarización menor (mayoritariamente los departamentos pertenecen a la categoría Medio-Bajo), exhibe un comportamiento más homogéneo. 
Gráfico 2. Región Centro: índice de bancarización. PCA espacial. Año 2010
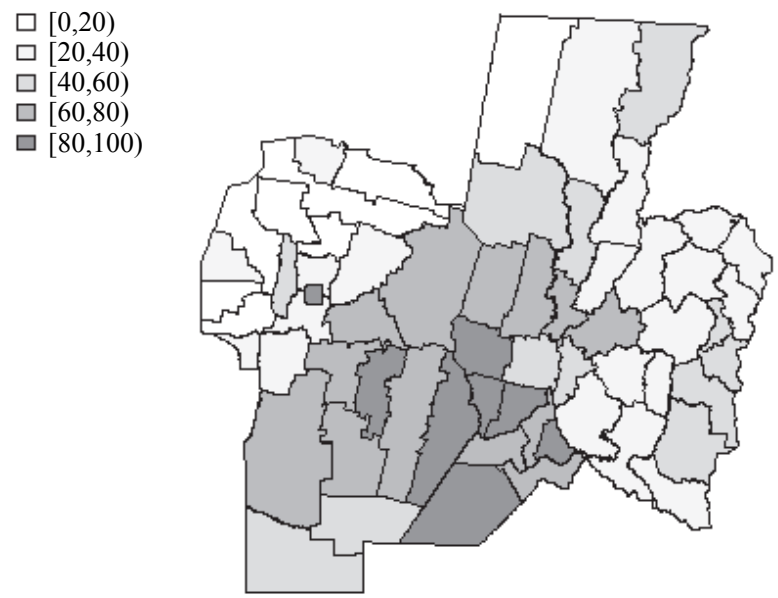

Fuente: elaboración propia.

Esta información puede complementarse con la tabla 5 que exhibe algunas medidas descriptivas calculadas para el IB obtenido a partir de PCA espacial para la región y discriminado por provincia, la que permite confirmar el ordenamiento realizado anteriormente.

Tabla 5. Índice de bancarización PCA espacial

\begin{tabular}{lcc}
\hline Provincia & Media & Coeficiente de variación (\%) \\
\hline Córdoba & 43,6 & 60,4 \\
Entre Ríos & 37,8 & 29,2 \\
Santa Fe & 60,8 & 39,5 \\
\hline Total & 47,3 & 50,7 \\
\hline
\end{tabular}

Fuente: elaboración propia.

\section{COMENTARIOS FINALES}

La bancarización y la inclusión financiera en los últimos años se han convertido en objetivos importantes a nivel internacional y en particular para la 
Argentina. En esta línea se puede mencionar una de las iniciativas más recientes del BCRA, la puesta en marcha el Plan Nacional de Bancarización Inclusiva 20152019 , el cual contempla un conjunto de acciones y medidas tendientes a garantizar una mayor inclusión financiera en el país. En este sentido, una medición adecuada de la evolución del proceso de bancarización resulta clave en la búsqueda de la inclusión financiera.

Con esta premisa, este estudio elabora un IB para la Región Centro para el año 2010 considerando aspectos referentes a las diferentes dimensiones de análisis: Magnitudes Agregadas, Disponibilidad y Cobertura Geográfica y Acceso y Utilización. El IB facilita la comprensión de la bancarización y contribuye a que exista un reconocimiento respecto de su trascendencia como elemento que puede apoyar al crecimiento y desarrollo económico, al considerar la dimensión espacial de los datos.

Se considera que el IB propuesto es un elemento importante para sintetizar e interpretar la bancarización de manera sencilla y constituye un insumo valioso para facilitar la toma de decisiones tanto de los hacedores de política (en materia de política económica y financiera) como de las instituciones financieras.

La construcción del IB utilizando datos georreferenciados, es decir asociados con una localización, resulta un aporte metodológico significativo, en tanto permite la medición de manera global y sintética al incorporar la dimensión espacial de los datos. Así, facilita una visión general de la bancarización en la Región Centro en su conjunto y en cada una de las provincias que la componen. Se destaca la provincia de Santa Fe, la cual exhibe un mayor nivel de bancarización. Sigue en importancia la provincia de Córdoba, pero con un comportamiento más heterogéneo que contrasta con el de la provincia de Entre Ríos, que, aunque presenta un nivel de bancarización menor, exhibe un comportamiento más homogéneo.

Pese a las limitaciones del índice, vinculadas fundamentalmente a la imposibilidad de contar con datos de indicadores parciales que midan en forma más adecuada las dimensiones Magnitudes Agregadas y Acceso y Utilización, este indicador debería ser considerado como una primera aproximación a la medición de la bancarización regional y, acaso, como un trabajo en proceso.

Finalmente, debe remarcarse la utilidad de medir el indicador a nivel de un área geográfica menor (localidad), estando limitada por la dificultad, cuando no la imposibilidad, de obtener información que permita medir a ese nivel los indicadores parciales con los que se construye el IB. 


\section{REFERENCIAS BIBLIOGRÁFICAS}

Acevedo Bohórquez, I., \& Velásquez Ceballos, E. (2008) Algunos conceptos de la econometría espacial y el análisis exploratorio de datos espaciales. Ecos de Economía, 12 (27), 9-34.

Amidzic, G., Massara, A., \& Mialou, A. (2014). Assessing Countries’ Financial Inclusion Standing: A new composite index. Washington: International Monetary Fund, Working Paper $N^{\circ} 14 / 36$.

Anastasi, A., Blanco, E., Elosegui, P., \& Sangiácomo, M. (2010). La bancarización y los determinantes de la disponibilidad de servicios bancarios en Argentina. Ensayos Económicos. Banco Central de la República Argentina, 60, 137-209.

Banco Central de la República Argentina (2010a). Información de Entidades Financieras Diciembre de 2010. Superintendencia de Entidades Financieras y Cambiarias. [Disponible en CD].

Banco Central de la República Argentina (2010b). Disponibilidades, Préstamos y Depósitos clasificados según la ubicación geográfica de la casa o sucursal de la entidad financiera.

Banco Central de la República Argentina (2015). Mi diccionario financiero. Recuperado de http://www.bancocentraleduca.bcra.gov.ar/PDFs/Diccionario_Financiero_Jovenes.pdf

Bivand, R., Altman, M., Anselin, L., Assunção, R., Berke, O., Bernat, A.,... \& Yu, D. (2010). spdep: Spatial dependence: weighting schemes, statistics and models. R package version 0.5-26.

Bivand, R., Altman, M., Anselin, L., Assunção, R., Berke, O., Bernat, A.,.. \& Yu, D. (2010). Spatial dependence: weighting schemes, statistics and models. [Software R Package versión 0.5-26] Recuperado de http://citeseerx. ist.psu.edu/viewdoc/download?doi=10.1.1.190.4891\&rep=rep1\&type=pdf

Cámara, N., \& Tuesta, D. (2014). Measuring Financial Inclusion: a multidimensional index. Madrid: BBVA Research, Working Paper $N^{\circ} 14 / 26$.

Castellanos, S., Castellanos, V., \& Flores, B. (2009). Factores de influencia en la localización regional de infraestructura bancaria. Economía Mexicana, 18 (2), 283-330.

Chakravarty, S., \& Pal, R. (2013). Measuring Financial Inclusion: an axiomatic approach. Journal of Policy modeling, 35 (5), 813-837.

Claessens, S. (2005). Access to Financial Services: a Review of the issues and Public Policy Objetives, World Bank Policy Research, Working Paper 3589. Recuperado de http://documents.worldbank.org/curated/ en/386121468140396062/pdf/wps3589.pdf

Djankov, S., Manraj, D., McLiesh, C., \& Ramalho, R. (2005). Doing Business Indicators: Why Aggregate, and How to Do It. Washington: World Bank. 
Domínguez Serrano, M., Blancas Peral, F. J., Guerrero Casas, F. M., \& González Lozano, M. (2011). Una revisión crítica para la construcción de indicadores sintéticos. Revista de Métodos Cuantitativos para la Economía y la Empresa, 11, 41-70.

Dray, S., \& Jombart, T. (2011). Revisiting Guerry's data: introducing spatial constraints in multivariate Analysis. The Annals of Applied Statistics, 5 (4), 2278-2299.

Chessel, D., Dufour, A. B., \& Thioulouse, J. (2004). The ade4 package-I- Onetable methods. $R$ News, 4 (1), 5-10.

Evanoff, D. (1988). Branch Banking and Service Accessibility. Journal of Money, Credit and Banking, 20 (2), 191-202.

García, F. (2017). Un índice único de bancarización con datos georreferenciados con una aplicación para la Argentina. Ecos de Economía, 21 (45), 24-38.

Geary, R. C. (1954). The contiguity ratio and statistical mapping. The Incorporated Statistician, 5 (3), 115-145.

Grupo de Monitoreo Macroeconómico (2011). Indicadores de bancarización. Buenos Aires. Recuperado de https://www.gmm-mercosur.org

Gupte, R., Venkataramani, B., \& Gupta, D. (2012). Computation of financial inclusion index for India. Procedia - Social and Behavioral Sciences, 37, 133-149.

Hasan, R., \& Islam, E. (2016). Financial Inclusion Index at district levels in Bangladesh: a distance-based approach. Bangladesh Bank, Working Paper Series No 1603. Recuperado de https://mpra.ub.uni-muenchen.de/71344/1/ MPRA_paper_71344.pdf

Herrera, M., Paz, J., \& Cid, J. (2012). Introducción a la Econometría Espacial. Una aplicación al estudio de la fecundidad en la Argentina usando $R$, University Library of Munich, MPRA Paper $N^{\circ} 41138$. Recuperado de https:// mpra.ub.uni-muenchen.de/41138/

Instituto Nacional de Estadísticas y Censos (2010). Censo Nacional de Población, Hogares y Viviendas 2010, procesado con Redatam+SP. Recuperado de http://www.indec.gov.ar.

Kumar, A. (2005). Access to Financial Services in Brazil. Directions in Development. Washington: The World Bank.

Lanzillotti, R., \& Saving, T. (1969). State branching restrictions and the availability of branching services: Comment. Journal of Money, Credit and Banking, 1 (4), 778-788.

Medina, C., \& Núñez, J. (2006). La Oferta de Servicios del Sector Financiero Formal en Bogotá. Universidad de los Andes, Centro de Estudios sobre Desarrollo Económico, Working Paper № 5. 
Mishra, R. N., Verma, P., \& Bose, S. (2014). Operationalising financial inclusion Index as a policy lever: Uttar Pradesh (in India) - A Case Study. Journal of Mathematics and Statistical Science, 2015, 149-165.

Moran, P. (1950). Notes on Continuous Stochastic Phenomena. Biometrika, 37 (1), 17-23.

Morales, L., \& Yáñez, A. (2006). La bancarización en Chile, concepto y medición. Superintendencia de Bancos e Instituciones Financieras de Chile, Serie Técnica de Estudios $\mathrm{N}^{\circ} 7$.

Nardo, M., Saisana, M., Saltelli, A., Tarantola, S., Hoffmann, A., \& Giovannini, E. (2008). Handbook on Constructing Composite Indicators: Methodology and User Guide. Paris: OECD Publishing.

Peña, D. (2002). Análisis de datos multivariantes. Madrid: Mc Graw.

Sarma, M. (2008). Index of financial inclusion. New Delhi: Indian Council for Research on International Economics Relations, Working Paper $\mathrm{N}^{\circ} 215$.

Sarma, M. (2012). Index of Financial Inclusion - A measure of financial sector inclusiveness. Berlin Working Papers on Money, Trade, Finance and Development, № 07.

Savage, D., \& Humphrey, D. (1979). Branching Laws and Banking Offices: Comment. Journal of Money, Credit and Banking, 11 (2), 227-230.

Seaver, W., \& Fraser, D. (1983). Branch Banking and the Availability of Banking Offices in Nonmetropolitan Areas. Atlantic Economic Journal, 11 (2), 72-78.

Seaver, W., \& Fraser, D. (1979). Banking and the availability of banking services in metropolitan areas. The Journal of Financial and Quantitative Analysis, 14 (1), 153-160.

Sethy, S. K. (2016). Developing a financial inclusion index and inclusive growth in India. Theoretical and applied economics, 2 (607), 187-206.

Schabenberger, O., \& F. Pierce. (2002). Contemporary statistical models for the plant and soil sciences. Boca Ratón, FL: CRC Press

Zulaica Piñeyro, C. M. (2013). Financial Inclusion index: proposal of a multidimensional measure for México. Revista Mexicana de Economía y Finanzas, 8 (2), 157-180.

(C) 2018 por los autores; licencia otorgada a la revista Estudios económicos. Este artículo es de acceso abierto y distribuido bajo los términos y condiciones de una licencia Atribución-No Comercial 3.0 Unported (CC BY-NC 3.0) de Creative Commons. Para ver una copia de esta licencia, visite http://creativecommons.org/ licenses/by-nc/3.0 
